\title{
Denture Essential Kit: An Introduction
}

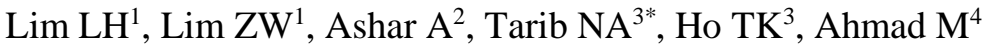 \\ 1. Faculty of Dentistry, Universiti Kebangsaan Malaysia, Jalan Raja Muda Abdul Aziz, 50300 Kuala Lumpur, Malaysia \\ 2. Department of Clinical Oral Biology, Faculty of Dentistry, Universiti Kebangsaan Malaysia, Jalan Raja Muda Abdul \\ Aziz, 50300 Kuala Lumpur, Malaysia \\ 3. Department of Prosthodontics, Faculty of Dentistry, Universiti Kebangsaan Malaysia, Jalan Raja Muda Abdul Aziz, \\ 50300 Kuala Lumpur, Malaysia \\ 4. Evercare Dental Practice, 43010 Kajang, Selangor, Malaysia
}

*E-mail: drtasyatarib@ukm.edu.my

\begin{abstract}
Good denture hygiene is an important component of oral health and in prevention of oral mucosal lesions. The strategies to provide impactful advice include denture education combined with a demonstration using a Denture Essential Kit (DEK).
\end{abstract}

Aim: To introduce DEK and to evaluate the effectiveness of denture hygiene delivery among denture wearers and clinicians.

Materials and Methods: A total of 78 patients were recruited in this study $(n=78)$. A set of questionnaire was given during denture delivery to assess the knowledge and habits on denture care before introduction of DEK. All patients were given denture hygiene in 3 methods: (i) verbally, (ii) brochure presentation and (iii) demonstration. Feedback among clinicians towards introduction of DEK was collected $(n=80)$.

Results: From 78 patients, 25 were first time denture wearers (32.1\%) while 53 were previous denture wearers $(67.9 \%)$. Out of $67.9 \%, 56.6 \%$ claimed that they had never received instructions on denture care during previous delivery stage. All patients showed significant effect on acknowledgment of receiving instructions on denture care $(\mathrm{p}<0.05)$. 99\% of clinicians agreed that introduction of DEK is useful while giving denture hygiene instructions.

Conclusion: Denture Essential Kit issued to patients during delivery appointment positively influenced their denture care perspective. Almost all clinicians agreed that DEK is beneficial to patient's overall health.

Keywords: denture care regime, denture cleanser, denture essential kit (DEK), denture hygiene

\section{Introduction}

The rehabilitative management of adults presented with partially dentate and complete edentulous conditions by prescribing removable partial dentures (RPDs) or complete dentures (CDs) are common management. Results of the National Oral Health Survey of Adult 2010 (NOHSA 2010) showed that $7.3 \%$ of subjects examined are affected with edentulism, with $6 \%$ of subjects had CDs. ${ }^{1}$ Removable prostheses are always the treatment of choice as they allow replacement of multiple missing teeth, able to restore function, speech, comfort and appearance, have the advantage of being able to remove to aid in cleaning and they are cost effective. ${ }^{2}$

However, there are some limitations associated with the use of removable prostheses which include compromised in term of stability, retention, support as compared to fixed prostheses such as bridge or implant and highly associated with oral diseases when inadequate of denture maintenance care and poor denture hygiene are noted. Good maintenance of denture and proper hygiene care can ultimately influence the success of rehabilitative treatment. Rehabilitative treatment is successful only when patients are aware of correct prostheses use and are motivated to take good care of denture hygiene. The quality of the denture fitting surfaces, occlusal relations, denture age, and hygiene are important factors contributing to the prevalence of oral mucosal lesions associated with denture use. ${ }^{3}$

A number of studies have highlighted the association between RPDs and an increased risk of periodontal disease, dental caries and denture stomatitis. ${ }^{2,}$ 4-5 They have concluded that RPDs act as plaque retentive factor in cases where satisfactory oral hygiene measures are 
lacking. Regular denture hygiene and care by denture wearers are important for good overall oral health and prevention of denture related oral lesions. ${ }^{6}$ Various denture hygiene procedures are mentioned in the literature and these can be divided into mechanical and chemical methods. ${ }^{7-8}$ A study conducted by Abhishek Apratim et al. concluded that most common method of cleaning was mechanical either with water or with soap. ${ }^{9}$ Frequency of cleaning with cleansing solutions was very low. Evidence has demonstrated that mechanical methods alone are insufficient to remove denture plaque and that chemical methods are also necessary. ${ }^{10-11}$

NOHSA 2010 review revealed that common problems experienced by denture wearers were discomfort due to loosen dentures, problems with either speech or chewing or both on wearing their dentures and soreness or pain on wearing dentures. ${ }^{1}$ Residual ridge resorption (RRR) is a common, lifelong condition that plagues complete edentulous patients. RRR occurs rapidly within 3 to 12 months after tooth removal, and continues throughout the patient's life. ${ }^{12-14}$ RRR compromises denture retention and stability, rendering the denture to be loose and nonserviceable to the patient. ${ }^{15}$ Patients with CDs required unique considerations due to their compromised oral anatomy, reduced adaptive capacity and systemic conditions or medications that further affect denture retention and stability. All of these factors reduced the patient's ability to successfully use their prostheses.16 In addition to hard and soft tissue alterations over time, these patients can also be affected by changes in quality or quantity of saliva due to medications or age, diminished bite force, and reduced neuromuscular control. ${ }^{17}$

Denture adhesive plays an important role in daily use of dentures. In 1997, Kelsey et al. evaluated the responses of experienced denture wearers with regards to the effectiveness of five denture adhesive pastes. ${ }^{18}$ The patients responded to questions about the effect of the pastes to quality and duration of retention as well as to mastication. Most of the subjects were satisfied with the use of denture adhesives for their masticatory performance and they agreed that denture adhesives increased their confidence in wearing denture. Kulak et al. in 2005 evaluated the subjective responses of denturewearers regarding effectiveness of two denture adhesive pastes. ${ }^{19}$ They reported that majority of patients were either fairly satisfied or very satisfied with retention of maxillary dentures. On the other hand, denture adhesives are a useful adjunct in denture prosthesis services, having specific roles in both the delivery appointment and postinsertion phases in immediate denture cases.

Proper denture hygiene care and denture maintenance are very much essential in maintaining the health of the oral cavity and dental prostheses. Therefore, it is essential that denture wearers are provided with instructions and knowledge on denture hygiene and maintenance when receiving their denture to ensure good oral health and longevity of remaining teeth and prostheses. A study by Barbosa et al. looked at patient knowledge of complete dentures on hygiene and care. They concluded that most of the patients evaluated in the study had little knowledge on cleaning methods. ${ }^{20}$ Many studies suggested a lack of awareness and little knowledge regarding denture hygiene and care among denture wearers. ${ }^{20-21}$ However, there is lack of current studies investigating how to improve patient awareness and knowledge regarding denture hygiene care and maintenance. Dental professionals have the obligations to motivate, instruct and advise patients on denture care and maintenance. The improvement of dental health and denture hygiene among denture wearers may be achieved through more effective education combined with a demonstration using a Denture Essential Kit (DEK) by dental professionals.

Thus, the aim of this study is to assess the attitude, knowledge and habits of denture wearers on denture hygiene and to evaluate effectiveness of denture hygiene delivery among denture wearers and clinicians with the use of DEK.

\section{Methodology}

\section{Ethical consideration}

Ethical approval was obtained from Research Ethics Committee, Medical Research and Industry Secretariat,Universiti Kebangsaan Malaysia Medical Committee [No: UKM 1.5.3.5/244/DD/2015/002(1)]. Informed consent was obtained by all patients.

\section{Selection of participants}

Patients treated by fourth and fifth year dental students, Faculty of Dentistry, Universiti Kebangsaan Malaysia (UKM) were recruited in this study. Only patients with RPDs, CDs and immediate dentures were enrolled in this study. Patients who required denture repair were excluded.

\section{Procedures}

All fourth and fifth year dental students were given DEK. DEK comprised of denture cleansing tablets, denture adhesive, denture brush and denture bath. An hour seminar was conducted to evaluate and demonstrate DEK to students by prosthodontists. Denture hygiene and application of denture adhesive were among the clinical demonstration given. To ensure students understand, they in return demonstrate the procedures. Denture essential pamphlets were prepared in 3 languages; Malay, Mandarin and English (Figure1).

A total of 78 patients were identified to be included in this study. They were given a set of questionnaire before the introduction of DEK during delivery appointment. The questionnaires were given to assess the attitude, knowledge and habits on denture hygiene and their awareness of its importance during delivery appointment. Following that, explanation and demonstration on DEK were given to patient by dental students. Feedback from clinicians with regards to introduction of DEK toward denture wearers was collected via questionnaire.

\section{Statistical analysis}

Data was collected and analyzed using Chi-square and McNemar test (SPSS version 23). 


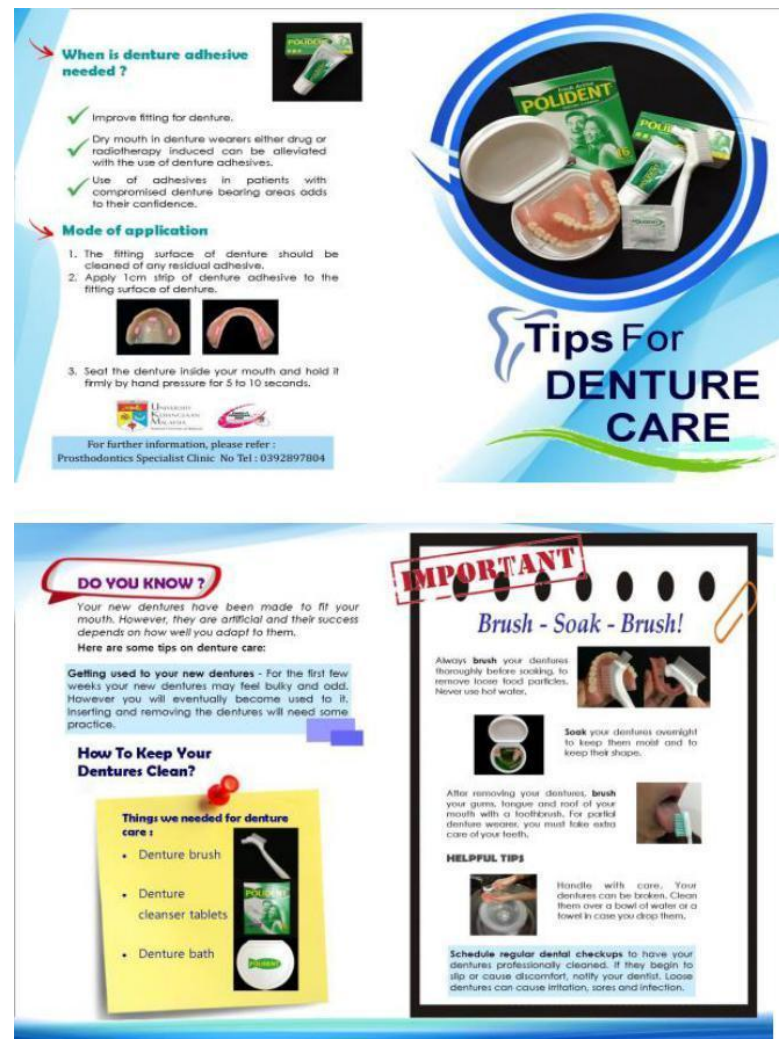

Figure 1. Denture essential pamphlet (English version)

\section{Results}

The demographic data of all enrolled participants is shown in Table 1. The age range of the patients was 22-87 years old with mean value of 61.71 years old. Most of the patients belonged to 61-70 (34.6\%) age group. Majority was previous denture wearer $(67.9 \%)$ and $63.6 \%$ had history of wearing denture more than 10 years.

Table 1. Demographic data of participants

\begin{tabular}{lll}
\hline Demographic & \multicolumn{2}{l}{ Total enrolled, $\mathbf{n = 7 8}$} \\
\hline Ethnicity: & 35 & $(44.9 \%)$ \\
Malay & 31 & $(39.7 \%)$ \\
Chinese & 10 & $(12.8 \%)$ \\
Indian & 2 & $(2.6 \%)$ \\
Others & 38 & $(48.7 \%)$ \\
Sex: & 40 & $(51.3 \%)$ \\
Male & & \\
Female & 25 & $(32.1 \%)$ \\
Type of Denture wearer: & 53 & $(67.9 \%)$ \\
First time &
\end{tabular}

According to the questionnaire, $56.6 \%$ of the previous denture wearers reported that they had never received any instructions from their previous dentist regarding how to take care of their denture, while $43.3 \%$ had received instructions from previous dentist but most of the instructions were in verbal form.

In terms of denture cleaning and care, $59.7 \%$ patients reported that they cleaned their dentures $2-4$ times daily. Among the denture wearers, majority of them (61\%) brushed their dentures with toothpaste (Figure 2). On the other hand, $59.6 \%$ of the patients soaked their dentures with water, $32.7 \%$ used cleansing tablets and $5.8 \%$ used other agents like salt or tea to soak their dentures (Figure 3 ). $4.2 \%$ of them encountered difficulty while cleaning their dentures and $16.7 \%$ do not remove their dentures before sleeping (Figure 4).

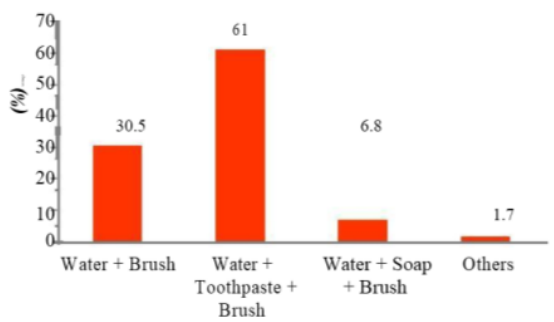

Figure 2. Agents used to brush dentures

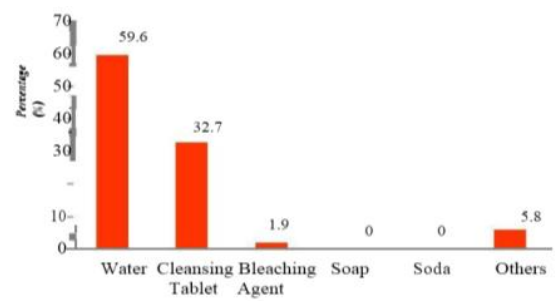

Figure 3. Agents used to soak dentures

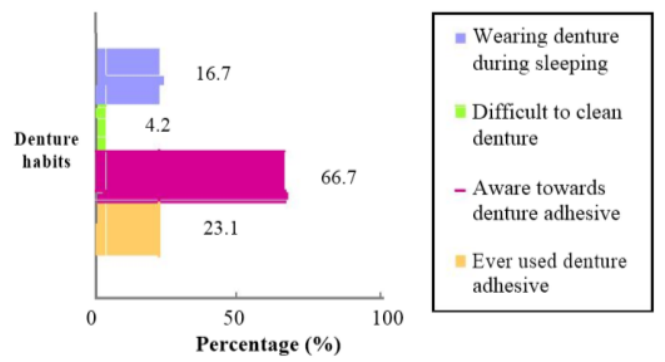

Figure 4. Denture wearer's denture habits and knowledge

Reasons of using denture adhesives are shown in Figure 5. Most of the patients did not encounter any problem while using denture adhesives $(42.8 \%)$ but some encountered problems such as difficult to remove adhesive paste (14.3\%), food traps (14.3\%) and unpleasant odor (9.5\%). The reasons of not using denture adhesives are shown in Figure 6. 


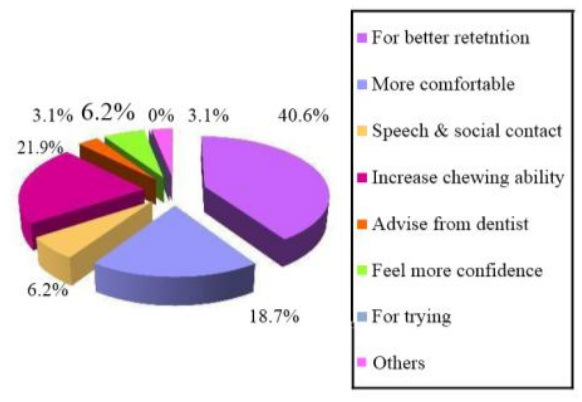

Figure 5. Reasons of using denture adhesive

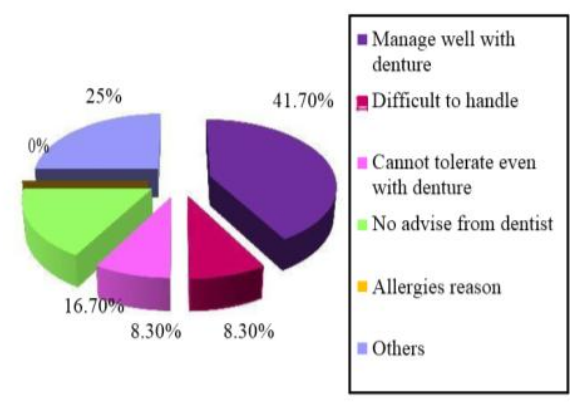

Figure 6. Reasons of not using denture adhesive

A total of 80 clinicians completed DEK feedback survey. Following introduction of DEK, majority clinicians showed positive responses with $99 \%$ clinicians agreed that DEK was useful while giving denture hygiene instructions combined with demonstration. Besides, most of the clinicians will use DEK (95.7\%) and recommend DEK to other practitioners in the future (100\%). $91.3 \%$ of clinicians believed that all practitioners should use DEK to give denture hygiene instructions while $16 \%$ of clinicians suggested the content/ material of existing DEK should include other cleansing aids such as interdental brush and floss for partially dentate patient.

Clinician's perspective on advantages of introduction of DEK in clinic is shown in (Figure 7).

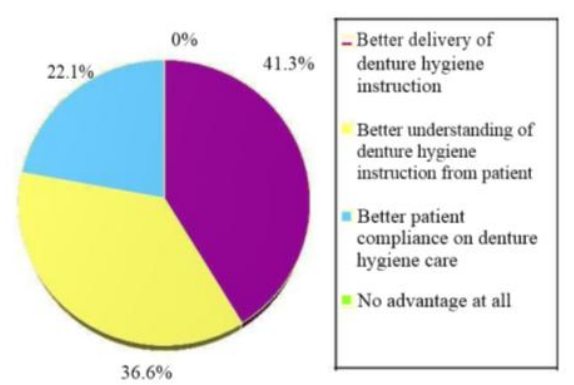

Figure 7. Clinician's perspective on advantages of introduction of DEK in clinic

A total of 27 patients acknowledged to receive DEK instructions and would change their habits in positive directions. This indicated that the observed tendency for those who acknowledged of receiving on denture care post introduction of DEK were statistically significant $(\mathrm{P}<0.05)$.

\section{Discussion}

Denture cleansing methods have been subject of research over the years. However, limited number of studies focused on the impact of DEK with the quality of denture wearing and care among denture wearers. This research introduced DEK which consist of denture cleansingtablets, denture adhesive, denture brush and denture bath to denture wearers and evaluated the effectiveness of denture hygiene delivery among denture wearers and clinicians.

In this study, most of previous denture wearers (56.6\%) reported that they have never received instructions on denture care. This finding was similar to other studies. ${ }^{22-25}$ This may be due to (i) dentists lack of knowledge on denture cleaning method and (ii) patients might not follow the instructions given by dentists. ${ }^{25}$ Majority of the previous denture wearers $(59.7 \%)$ reported that they cleaned their denture 2 to 4 times daily. This frequency was higher than study by Dikbas et al. where $25 \%$ of individuals reported that they cleaned their dentures 3 times daily. ${ }^{24}$ However, some studies have shown higher frequency of cleaning as Peracini et al. who reported $73.58 \%$ cleaned their dentures 3 or more times daily and Pietrokvoski et al. reported that $96 \%$ of patients reported cleaning their dentures 2 or more times per day. ${ }^{23,26}$

Previous study by Hoad-Reddick et al. reported that a combination of methods (brushing and soaking) was used more frequently. ${ }^{22}$ Veres et al. also found majority of patients $(59 \%)$ brushed and immersed their dentures. ${ }^{27}$ In the present study, $72.6 \%$ of the patients cleaned their dentures with both brushing and soaking method. Ideally, the combination of brushing and soaking method has been recommended as the effective way for cleaning dentures due to a better plaque control can be achieved. ${ }^{28}$ In this study, almost all patients $(98.3 \%)$ used brushing method for denture cleansing. The result found to be similar with previous studies by Peracini et al. (100\%) and Milward P et al. (72.4\%). ${ }^{23,2}$ In the study by Veres et al., $70 \%$ of the patients used dentifrice to clean their dentures. ${ }^{27}$ Brushing with dentifrice was performed by $61 \%$ of the patients in this study, which also found to be same with the finding from a previous study. ${ }^{23}$ This could be due to dentifrice has the advantage of being simple to use and relatively inexpensive. However, if used with an improper brushing technique, dentifrice can damage the prosthesis material due to the potential abrasive wear of the denture material. ${ }^{24,} 28$ In addition, brushing with toothpaste may make denture surface rougher, which increases the accumulation of dental plaque and reduces the shine of denture surfaces. ${ }^{29}$

From the respond, $59.6 \%$ patient soaked their denture in water and the findings almost similar to the study by Baran and Nalcaci. ${ }^{30}$ Furthermore, a small percentage of patients $(32.7 \%)$ used denture cleansing tablets to soak their dentures. But this habit was positively higher than the previous studies that found that $10.40 \%$ and $2.67 \%$ of patients soaked their dentures with denture cleansing tablets. $^{22,3}$ This could be due to lack of awareness 
regarding cleansing tablets and unaffordable cost for some of the patients. ${ }^{3}$

In the present study, $66.7 \%$ stated that they are aware of denture adhesive. The awareness toward denture adhesive is lower compared to previous studies as $100 \%$ reported by G. L. Polyzois and $87 \%$ by Özcan et al. ${ }^{31,32}$

The purpose of denture adhesives is to benefit denture wearers with a more appropriate fit and comfort and improve their chewing ability and confidence. ${ }^{32}$ However, only $23.1 \%$ of the patients used denture adhesive in this study. $40.6 \%$ of patients used denture adhesive for better denture retention which is relatively similar to previous study by G. L. Polyzois and C. de Baat. ${ }^{31}$

This study had shown that $83.3 \%$ of patients removed their dentures at night and this habit was positively higher than other studies. ${ }^{24,} 33$ Ideally prostheses should not be worn overnight to give rest for the supporting tissues. ${ }^{22}$

\section{Conclusions}

This study has provided information regarding the knowledge and denture care habits among denture wearers. Introduction of DEK to patients during delivery appointment had positively influenced their denture care perspective. Results of this study had reflected a lack of knowledge and correct denture care habits among denture wearers attending dental clinic in UKM. Clinicians found that DEK helps in easy delivery of denture hygiene instruction. Feedbacks from clinician towards introduction of DEK can serve as basic information for future study.

\section{Acknowledgment}

Sincere thanks to GlaxoSmithKline (GSK) Healthcare Company for their sponsorship in providing Denture Essential Kits to carry out this study.

\section{References}

1. National Oral Health Survey of Adults (NOHSA). Ministry of Health Malaysia. 2010. Project reference No: NMRR-09388-3480.

2. Milward P, Katechia D, Morgan MZ. Knowledge of removable partial denture wearers on denture hygiene. $\mathrm{Br}$ Dent J. 2013;215:E20-E20.

3. Patel IB, Madan G, Patel B, Solanki K, Chavda R. Behaviours and hygiene habits of a sample population of complete denture wearers in Ahmedabad. J IntOral Health. 2012;4(2):29-38

4. Budtz-Jorgensen E, Bertran U. Denture stomatitis I. The etiology in relation to trauma and infection. ActaOdontol Scand. 1970;28(1):71-92.

5. Schou L, Wight C, Cumming C. Oral hygiene habits, denture plaque, presence of yeasts and stomatitis in institutionalised elderly in Lothian, Scotland. Community Dent Oral Epidemiol. 1987;15(2):85-89.

6. Budtz-Jørgensen E. Oral mucosal lesions associated with the wearing of removable dentures. J OralPathol 1981;10(2):65-80.

7. Paranhos HF, Silva-Lovato CH, Venezian GC, Macedo LD De Souza RF. Distribution of biofilm on internal and external surfaces of maxillary complete dentures: the effect of hygiene instruction. Gerodontology. 2007;24(3):162-168.
8. Paranhos HF, Silva-Lovato CH, Souza RF, Cruz PC, Freitas KM, Peracini A. Effects of mechanical and chemical methods on denture biofilm accumulation. JOral Rehabil. 2007;34(8):606-612.

9. Apratim A, Shah SS, Sinha M, Agrawal M, Chhaparia N, Abubakkar A. Denture hygiene habits among elderly patients wearing complete dentures. JContemp Dent Pract. 2013;14(6): 1161-1164.

10. Mahonen K, Virtanen K, Larmas M. The effect of prosthesis disinfection on salivary microbial levels. JOral Rehabil. 1998;25(4):304-310.

11. Dills SS, Olshan AM, Goldner S, Brogdon C. Comparison of the antimicrobial capability of an abrasive paste and chemical soak denture cleaners. JProsthet Dent. 1988;66(4):467-470.

12. Atwood DA, Coy WA. Clinical, cephalometric, and densitometric study of reduction of residual ridges. JProsthet Dent. 1971;26(3):280-295.

13. Atwood D. Bone loss of edentulous alveolar ridges. JPeriodont. 1979;50(4s):11-21.

14. Tallgren A. The continuing reduction of the residual alveolar ridges in complete denture wearers: A mixedlongitudinal study covering 25 years. JProsthet Dent. 1972;27(2):120-132.

15. McCartney JE. Prosthetic problems resulting from facial and intraoral changes in the edentulous patient. J Dent. 1981;9(1):71-83

16. Atwood DA. Reduction of residual ridges: A major oral disease entity. J Prosthet Dent. 1971;26(3):266-279.

17. Felton DA. Edentulism and comorbid factors. JProsthodont. 2009;18(2):88-96.

18. Kelsey CC, Lang BR, Wang RF. Examining patients' responses about the effectiveness of five denture adhesive pastes. J Am Dent Assoc. 1997;128(11):1532-1538

19. Kulak Y, Ozcan M, Arikan A. Subjective assessment by patients of the efficiency of two denture adhesive pastes. J Prosthodont. 2005;14(4):248-252.

20. Barbosa LC, Ferreira MRM, de Carvalho FC, Lauria RA, de Lemos LMC, Viana AC. Edentulous patients' knowledge of dental hygiene and care of prostheses. Gerodontology. 2008;25(2):99-106.

21. Cakan U, Yuzbasioglu E, Kurt H, Kara HB, Turunc R, Akbulut A, Aydin KC. Assessment of hygiene habits and attitudes among removable partial denture wearers in a university hospital. Niger J Clin Pract. 2015;18(4):511-515.

22. Hoad-Reddick G, Grant A, Griffits CS. Investigation into the cleanliness of dentures in elderly population. J Prosthet Dent. 1990;64(1):48-52.

23. Peracini A, Andrade IM, Paranhos Hde F, Silva CH, de Souza RF. Behaviors and hygiene habits of complete denture wearers. Braz Dent J. 2010;21(3):247-252

24. Dikbas I, Koksal T, Calikkocaoglu S. Investigation of the cleanliness of dentures in a university hospital. IntJ Prosthodont. 2006;19(3):294-298.

25. Nevalainen MJ, Narhi TO, Ainamo A. Oral mucosal lesions and oral hygiene habits in the homeliving elderly. J Oral Rehabil. 1997;24(5):332-337.

26. Pietrokovski J, Azuelos J, Tau S, Mostavoy R. Oral findings in elderly nursing home residents in selected countries. Oral hygiene conditions and plaque accumulation on denture surfaces. J Prosthet Dent. 1995;73(2):136-141.

27. Veres EM, Wolfaardt JF, Hnizdo E. Denture cleansers: Part III - A survey of materials and methods employed by denture wearers. J Dent Assoc SAfr. 1985;40(10):591-594.

28. Jagger DC, Harrison A. Denture cleansing - the best approach. Br Dent J. 1995;178(11):413-417.

29. Tanoue N, Matsumura H, Atsuta M. Wear and surface roughness of current prosthetic composites after toothbrush/dentifrice abrasion. J Prosthet Dent. 2000;84(1):93-97.

30. Baran I, Nalçaci R. Self-reported denture hygiene habits and oral tissue conditions of complete denture wearers. Arch Gerontol Geriatr. 2009;49(2):237-241.

31. Polyzois GL, de Baat C. Attitudes and usage of denture adhesives by complete denture wearers : a survey in Greece and the Netherlands. Gerodontology. 2012;29(2):e807e814. 
32. Özcan M, Kulak Y, Arikan A, Silahtar E. The attitude of complete denture wearers towards adhesives in Istanbul. J Oral Rehabil. 2004;31(2):131-134.

33. Marchini L, Tamashiro E, Nascimento DFF, Cunha VPP. Self-reported denture hygiene of a sample of edentulous attendees at a University dental clinic and the relationship to the condition of the oral tissues. Gerodontology. 2004;21(4):226-228 